\title{
Implantable cardioverter defibrillator in non-ischemic cardiomyopathy: a meta-analysis of randomized controlled trials
}

\author{
Ahmad Masri ${ }^{1}$, Muhammad Hammadah ${ }^{2}$, Evan Adelstein ${ }^{1}$, Sandeep Jain ${ }^{1}$, Samir Saba ${ }^{1}$ \\ ${ }^{1}$ Heart and Vascular Institute, University of Pittsburgh Medical Center, Pittsburgh, PA, USA; ${ }^{2}$ Division of Cardiology, Department of Medicine, \\ Emory University, Atlanta, Georgia, USA \\ Contributions: (I) Concept and design: A Masri, M Hammadah, S Saba; (II) Administrative support: A Masri; (III) Provision of study materials or \\ patients: A Masri, M Hammadah, S Saba; (IV) Collection and assembly of data: A Masri, M Hammadah; (V) Data analysis and interpretation: A \\ Masri, M Hammadah, S Saba; (VI) Manuscript writing: All authors; (VII) Final approval of manuscript: All authors. \\ Correspondence to: Samir Saba, MD. Heart \& Vascular Institute, University of Pittsburgh, Medical Center, 200 Lothrop Street, PUH, Suite B535, \\ Pittsburgh, Pa 15213, USA. Email: Sabas@upmc.edu.
}

\begin{abstract}
Background: Sudden cardiac death (SCD) is a significant cause of mortality in patients with non-ischemic cardiomyopathy (NICM). Implantable cardioverter defibrillators (ICDs) are currently indicated for the primary prevention of SCD in these patients. Conflicting results from published randomized controlled trials (RCTs) have recently questioned the protective role of ICD in NICM patients to perform an updated metaanalysis of the effect of ICDs on outcomes of NICM patients.

Methods: We performed a search of PubMed, EMBASE, and Cochrane databases for RCTs comparing ICD to medical therapy in patients with NICM. Outcomes were all-cause mortality, SCD, and cardiac mortality. Mantel-Haenszel (MH) risk ratios (RR) were calculated using random-effects meta-analysis for the outcomes mentioned. Heterogeneity was assessed using $\mathrm{I}^{2}$ statistics and Q-statistic.

Results: A total of five RCTs met the inclusion criteria, with a total of 1,503 patients in the ICD group and 1,364 patients in the medical therapy. Compared to medical therapy, ICD use resulted in a $24 \%$ relative risk reduction (RRR) of all-cause mortality (RR 0.76, 95\% CI 0.64-0.91, P=0.002), and 60\% RRR of SCD (RR 0.40, 95\% CI 0.18-0.90, $\mathrm{P}=0.03$ ), but was not associated with a statistically significant benefit in the prevention of cardiac mortality (RR 0.75, 95\% CI 0.39-1.44, P=0.39). Limiting the analysis to patients who received an ICD only without cardiac resynchronization therapy (CRT) pacemakers (4 trials, 1,825 patients) showed similar allcause mortality results with $24 \%$ RRR in ICD group (RR 0.76, 95\% CI 0.61-0.94, P=0.01).
\end{abstract}

Conclusions: ICD therapy reduces all-cause mortality and SCD in patients with NICM.

Keywords: Death; non-ischemic cardiomyopathy (NICM); implantable defibrillators; meta-analysis; randomized controlled trial (RCT)

Submitted Apr 12, 2017. Accepted for publication Jun 02, 2017.

doi: 10.21037/cdt.2017.06.06

View this article at: http://dx.doi.org/10.21037/cdt.2017.06.06

\section{Introduction}

Non-ischemic cardiomyopathy (NICM) is broadly defined as any cardiomyopathy in which there is no evidence of obstructive coronary artery disease that could explain the degree of left ventricular dysfunction. Although it is challenging to determine the exact prevalence of NICM, prior reports suggest that it is about 36 in 100,000 (1).
Sudden cardiac death (SCD) is a significant cause of death in the heart failure with reduced ejection fraction population, as demonstrated by the Framingham Heart study where the presence of heart failure led to a fivefold increase in the risk of SCD (2).

Five randomized clinical trials (RCTs) evaluating the role of implantable cardioverter defibrillator (ICD) therapy on survival of NICM patients have been published over 
the past 2 decades (3-7). Of these, three studies enrolled NICM patients exclusively $(3,5,6)$, while the other two studies included both patients with ICM and NICM $(4,7)$. The Sudden Cardiac Death in Heart Failure Trial (SCD$\mathrm{HeFT}$ ) demonstrated benefit from ICD therapy in reducing all-cause mortality in the overall cohort of patients and a trend in the same direction when the NICM patients were examined separately (7). The Comparison of Medical Therapy, Pacing, and Defibrillation in Heart Failure (COMPANION) trial demonstrated that ICD therapy when combined with cardiac resynchronization therapy (CRT) also reduces the risk of death (4). Based primarily on the results of the SCD-HeFT trial, the American College of Cardiology/American Heart Association (ACC/ AHA) guidelines (class IA) and the European Society of Cardiology (ESC) guidelines (class IB) recommend ICD therapy in symptomatic NICM patients with a left ventricular ejection fraction (LVEF) $\leq 35 \%(8,9)$. More recently, the Danish Study to Assess the Efficacy of ICDs in Patients with Non-ischemic Systolic Heart Failure on Mortality (DANISH) trial (5) demonstrated that, compared to medical therapy alone or with CRT pacing, ICDs did not reduce all-cause mortality but reduced SCD (5). The DANISH trial shed some doubt again on the value of ICD therapy in reducing mortality in NICM patients.

Given these mixed results and the concern that each RCT alone was underpowered, we performed a systematic review and a meta-analysis of the role of ICDs in the primary prevention of all-cause mortality, SCD, and cardiac death in patients with NICM.

\section{Methods}

We performed a meta-analysis of all the RCTs that assessed the role of ICDs in primary prevention of death in patients with NICM, following PRISMA (Preferred Reporting Items for Systematic reviews and Meta-Analyses) guidelines (10). We searched three databases, including PubMed, EMBASE, and Cochrane, for relevant studies from Jan 1980 to Aug 2016. This search was carried independently by two investigators (A.M and M.H). We included the following key terms: NICM and ICDs, NICM and SCD, NICM and CRT, and NICM and primary prevention. Also, for each of these search terms, NICM was substituted for idiopathic dilated cardiomyopathy. The search was limited to RCTs and English language only. Full texts and references of potential studies were reviewed.

RCTs that compared ICDs versus standard medical therapy for the prevention of SCD in NICM patients were included. We included trials that evaluated only NICM patients and trials that had mixed NICM and ICM patients if mortality was specifically reported in the NICM group. For the purpose of this analysis, only outcome results of NICM patients were considered.

Studies that were observational, involving only ICM patients, having Amiodarone as the comparator group or those published in non-English language were excluded. Qualities of each individual trial were assessed based on description of the randomization process, the follow up process, description of drop-outs or withdrawals, and description of crossovers. Given that the ICD arm is a procedural arm, we did not assess RCTs for blinding.

Relevant data were collected independently by two investigators (A.M and M.H). This included the number of participants randomized, duration of follow up, age, comorbidities, New York Heart Association (NYHA) class, mean LVEF, medications at baseline and during follow-up, concurrent use of other devices including CRT pacemakers, rates of all-cause death, and SCD, cardiac death. The primary outcome of interest was all-cause mortality in patients who received ICDs vs. who were on medical therapy. Secondary outcomes included SCD, cardiac death, and death secondary to cardiac causes after excluding SCD.

\section{Statistical analysis}

Continuous variables are expressed as mean. Categorical data are expressed as percentage. Using summary statistics, we report Mantel-Haenszel (MH) risk ratio (RR) using DerSimonian and Laird's random-effect models (11). Statistical significance was set at a $\mathrm{P}$ value $<0.05$ (2-tailed). Inter-study heterogeneity was assessed with the inconsistency index $\left(\mathrm{I}^{2}\right)$ statistic (range $0 \%$ to $100 \%$, we used $\mathrm{I}^{2}>60 \%$ as a cutoff for significant heterogeneity) and $\mathrm{Q}$-statistic derived from chi-square test ( $\mathrm{P}$ value cut-off $<0.1$ for heterogeneity) (12). These measures of heterogeneity are reported on the Forest plot of each analysis. Given the lack of patient level data, we used summary statistics for each trial when available by using the reported Hazard Ratios as time-to-event. Individual studies risk estimates were combined using the inverse variance weighted averages of logarithmic RRs in the random-effects model. Potential publication bias was evaluated by Begg's funnel plot (13).

In order to detect and analyze any potential heterogeneity, sensitivity analyses were performed through removing one study at a time and assessing its effect on 


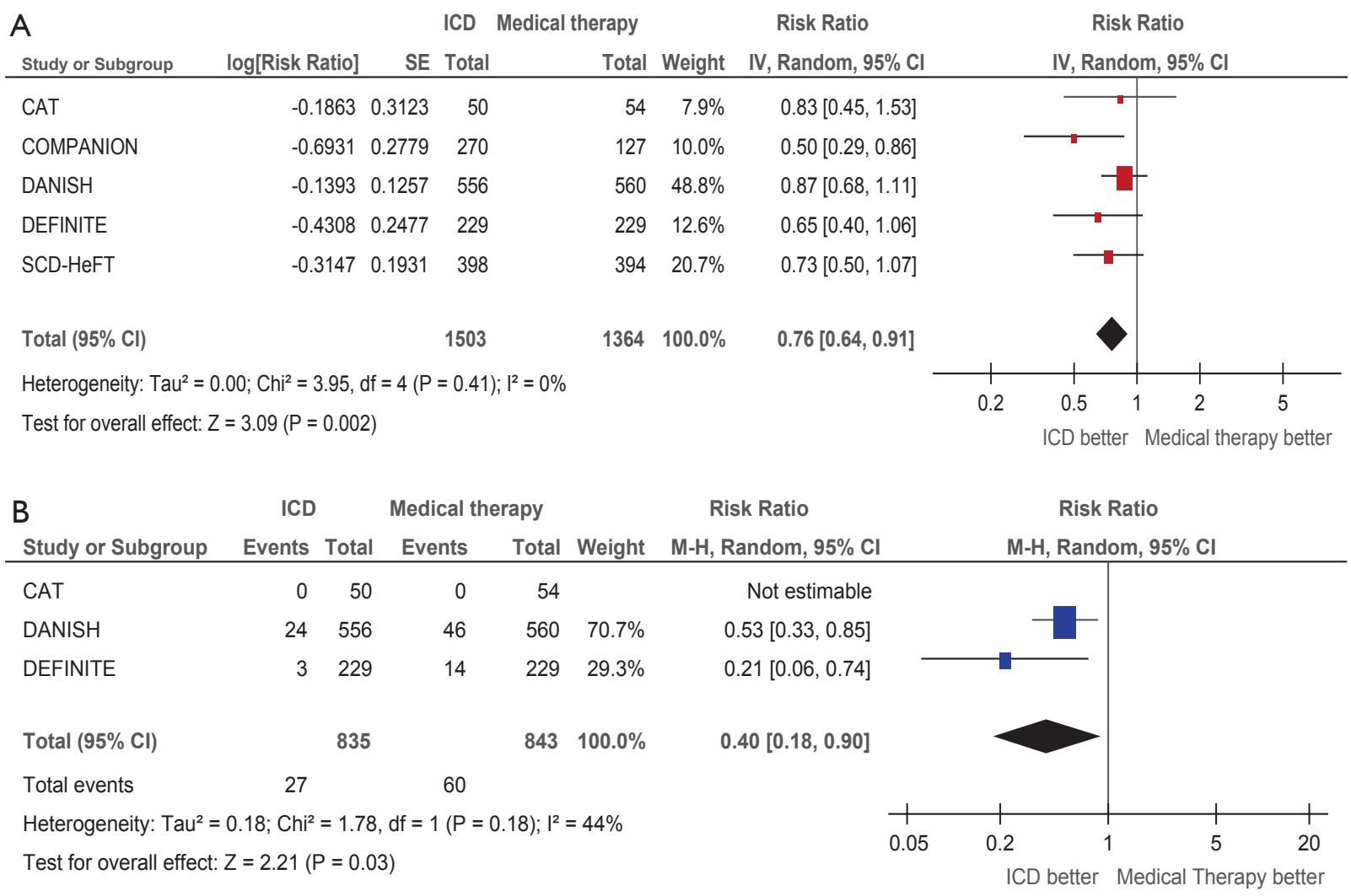

Figure 1 Forest plots comparing internal cardioverter defibrillator versus medical therapy. (A) all-cause mortality (B) sudden cardiac death.

the outcome in each individual analysis done. To further understand the effect of varying degrees of follow up on the primary outcome, we performed a sensitivity analysis by dividing the studies into two groups; those with a short period of follow up $(\leq 3$ years; CAT, DEFINITE, and COMPANION) or longer follow up ( $>3$ years; DANISH and SCD-HeFT). All analyses performed using Review Manager Version 5.3 (The Cochrane Collaboration, Oxford, England).

\section{Results}

\section{Study selection}

The initial search yielded 219 studies. After screening, 15 studies were selected based on study title, and their full texts were reviewed. Ten studies were excluded (6 were done in ICM, 3 secondary prevention trials in NICM, and 1 trial used Amiodarone as the comparator group). Five RCTs were included in the final analysis (Figure S1, online supplement). Heterogeneity analyses showed that although these 5 RCTs had differences in their study design and specific inclusion criteria, all of them reported similar outcome points for NICM patients and were homogeneous enough to be incorporated in the meta-analysis. Specific heterogeneity measures are reflected in the respective Forest plots (Figures 1,2).

\section{Qualitative analysis of the included RCTs}

The 5 RCTs enrolled a total of 2,867 patients with NICM who were randomized to ICD \pm CRT $(n=1,503)$ or medical therapy \pm CRT $(\mathrm{n}=1,364)$. The individual characteristics of each RCT are summarized in Table S1. The outcomes reported in each individual RCT are summarized in Table 1. For the SCD-HeFT and COMPANION trials, the outcome of all-cause mortality included in this metaanalysis was reported specifically for NICM patients in the original publication. However, the individual characteristics of both trials summarized in Table $S 1$ are reflective of the overall population of both ICM and NICM. Also, the SCDHeFT trial, we only report on the outcome of patients 


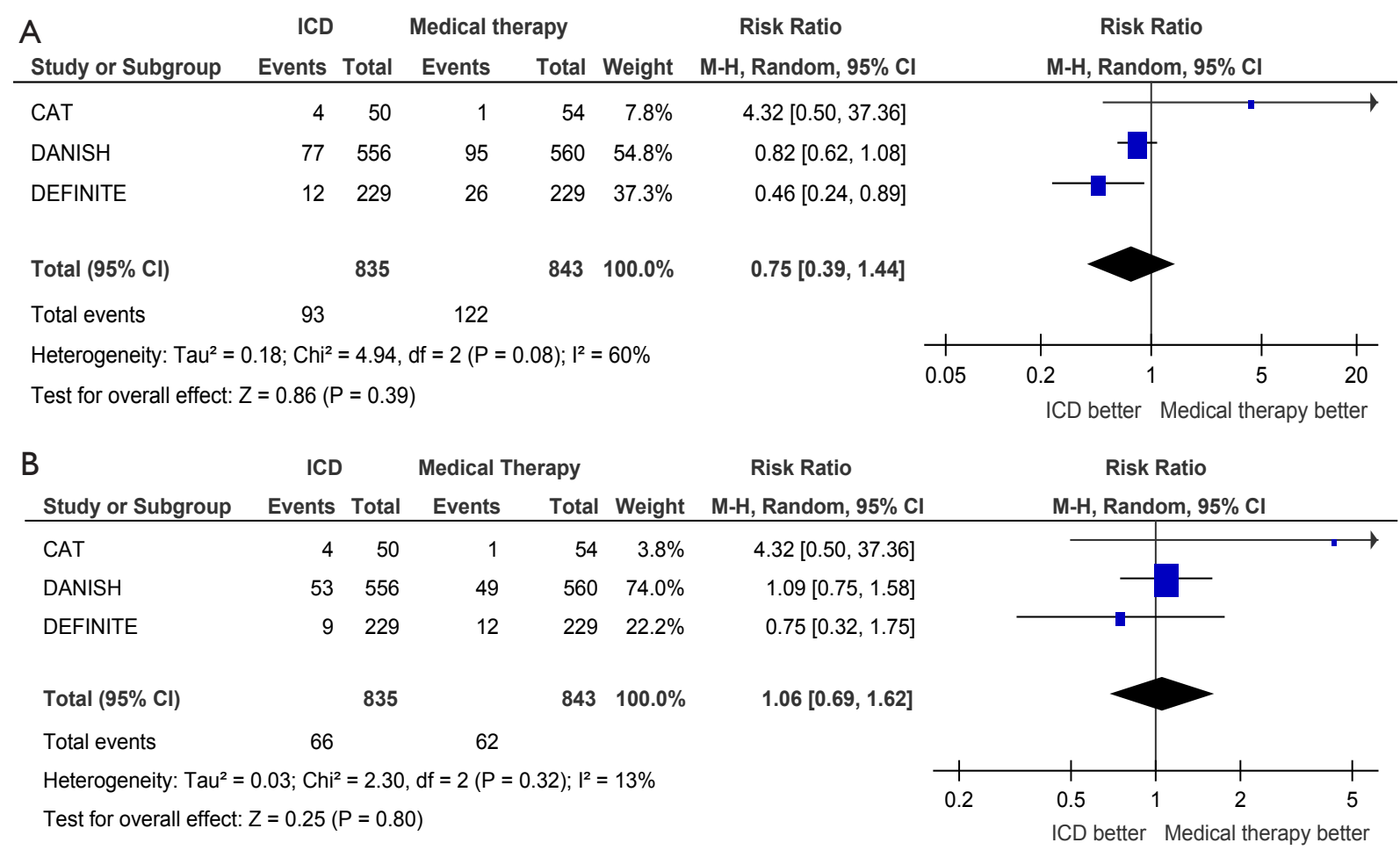

Figure 2 Forest plots comparing internal cardioverter defibrillator versus medical therapy (A) cardiac mortality (B) cardiac mortality excluding cases of sudden cardiac death.

implanted with an ICD as compared to medical therapy, and we excluded the patients assigned to the Amiodarone group. For the COMPANION trial, the outcome of the CRT pacemaker only group is not included.

The general design of all of these RCTs was similar (3-7). Patients were randomized into an ICD arm (with only the DANISH and the COMPANION trials having additional CRT pacemakers inserted) or into a medical therapy arm. Except for the recent DANISH trial, all other RCTs enrolled patients from 1991 to $2002(3,4,6,7)$. Mean LVEF ranged from $20 \%$ to $25 \%$ in all RCTs $(3-7)$. The majority of patients enrolled had NYHA class II-III; only the DEFINITE trial included patients with NYHA class I. The COMPANION and DANISH trials included patients with NYHA class IV. However, in the DANISH trial, all NYHA class IV patients received CRT pacing and they represented a very small minority of the overall DANISH population (1\%).

In terms of CRT pacing, the medical therapy arm of the COMPANION trial did not include CRT devices whereas the medical arm of the DANISH trial, had about $58 \%$ patients with CRT pacing devices. In all the other RCTs, the mean QRS duration was either $<120$ millisecond $(3,6)$ or not reported (7) (Table S1). In the DANISH trial, patients in the medical therapy and ICD arms were also stratified according to CRT implantation status; survival was not statistically different in the ICD group as compared to medical therapy in patients who had CRT device implanted (HR 0.91, 95\% CI 0.64-1.29, $\mathrm{P}=0.59$ ) or those who did not (HR 0.83, 95\% CI 0.58-1.19, $\mathrm{P}=0.31$ ).

Given that standard medical therapy for heart failure in patients with NICM has been shown to affect survival, all five RCTs reported on guideline directed medical therapy, including Angiotensin-converting-enzyme inhibitors (ACEIs), Angiotensin II receptor blockers (ARBs), Beta-blockers, and Mineralocorticoid receptor antagonist (MRA) (3-7). All RCTs had $>90 \%$ utilization rate of ACE-Is or ARBs. The Beta-blocker utilization rate was however more variable ranging between $3.7 \%$ and $92 \%$, based on how recent the study is (i.e., due to changes in guidelines' directed medical therapy). Only the DEFINITE and DANISH trials had $>80 \%$ utilization rate of beta-blockers. For MRA, rates of utilization were low $(<21 \%)(7)$, not reported $(3,6)$, or ranged between $55 \%$ and $59 \%$ for the COMPANION and DANISH trials. 


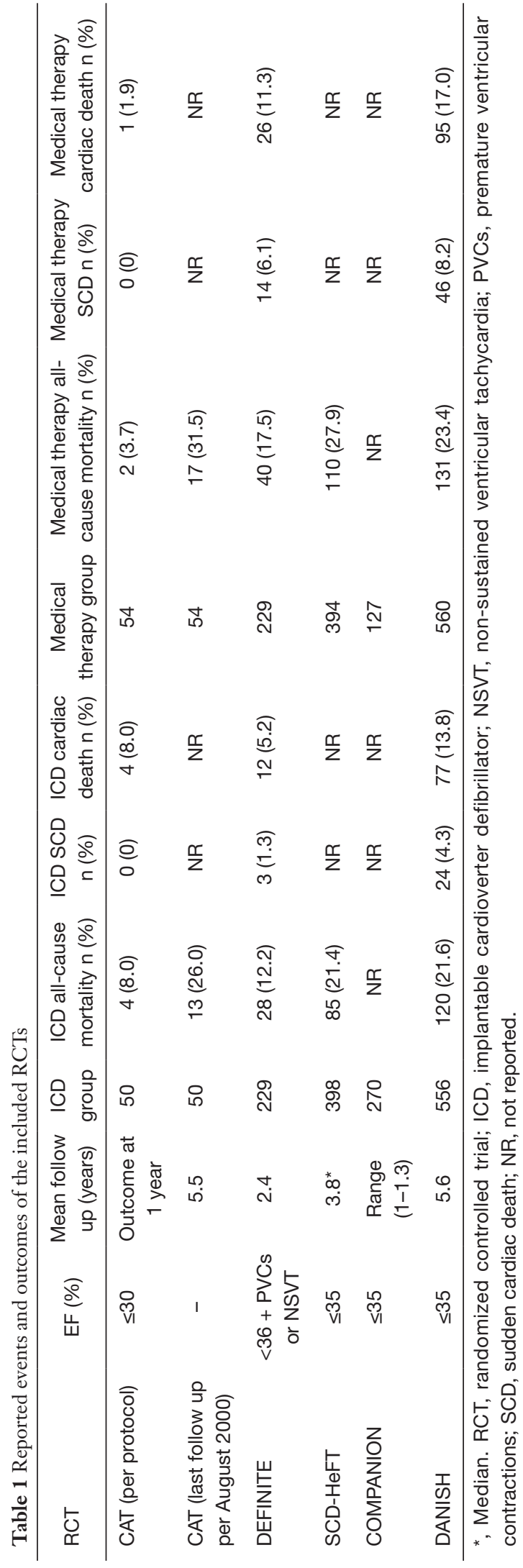

Crossover was reported in 3 out of 5 RCTs (4-6). Crossover to the ICD arm was $26 \%$ in the COMPANION trial, $10 \%$ in the DEFINITE trial, and $4.8 \%$ in the DANISH trial.

\section{Quantitative analysis of the included RCTs}

\section{All-cause mortality}

All 5 RCTs included in this analysis reported the primary outcome of all-cause mortality. Pooled analysis demonstrates that ICD therapy significant reduces all-cause mortality by $24 \%$ to standard medical therapy (RR 0.76 , 95\% CI 0.64-0.91, $\mathrm{P}=0.002$, Figure $1 A$ ). Sensitivity analysis was performed through removing one RCT at a time (five analyses were done) and studying the change of the pooled estimate. The statistical significant benefit of ICDs in primary prevention of all-cause mortality persisted in all analyses, even with the exclusion of SCD-HeFT trial (RR $0.74,95 \%$ CI $0.58-0.95, \mathrm{P}=0.02)$. Funnel plot is shown in Figure S2 (online supplement).

In order to further understand the effect of CRT on all-cause mortality, a sensitivity analysis was performed in which the overall survival HR in the DANISH trial was substituted for the HR comparing patients with ICD vs. medical therapy who have not received a CRT device. The benefit of ICDs persisted, with a relative risk reduction (RRR) of $28 \%$ (RR $0.72,95 \%$ CI $0.59-0.88, \mathrm{P}=0.001$ ); Figure $S 3$ (online supplement). Then, the COMPANION trial data were removed to have all four RCTs (including DANISH) with no patients who have received CRT device that could have confounded the outcome. However, the results persisted, with a RRR of $24 \%$ (RR $0.76,95 \%$ CI 0.61-0.94, P=0.01), Figure S4 (online supplement). Limiting the analysis to studies with follow up $\leq 3$ years did not change the results: RR 0.64 (95\% CI $0.47-0.87, \mathrm{P}=0.004$ ). Further, limiting the analysis to only DANISH and SCDHeFT (follow up $>3$ years) resulted in decreasing the magnitude of effect of ICD treatment which is driven by the DANISH trial (weight 70.2\%): RR 0.83 (95\% CI 0.67-1.02, $\mathrm{P}=0.07)$.

\section{SCD}

Three RCTs reported on SCD $(3,5,6)$. There were 27 SCD out of 835 patients who received ICDs vs. 60 SCD out of 843 patients who received medical therapy. The pooled summary estimate shows a statistically significant $60 \%$ RRR of SCD in patients undergoing ICD implantation as compared to medical therapy (RR $0.40,95 \%$ CI $0.18-0.90$, 
$\mathrm{P}=0.03$ ). If this analysis is confined to the two studies that reported hazard ratios of SCD (DEFINITE and DANISH trials), ICD therapy remains protective against SCD with a $62 \%$ RRR over medical therapy (RR $0.38,95 \%$ CI $0.16-$ $0.86, \mathrm{P}=0.02)$.

\section{Cardiac death}

Three RCTs reported specific events related to cardiac death including cardiac death secondary to $\operatorname{SCD}(3,5,6)$. There were 93 cardiac deaths out of 835 patients who received ICDs vs. 122 cardiac deaths out of 843 patients who received medical therapy, with a pooled RR 0.75 (95\% CI $0.39-1.44, \mathrm{P}=0.39$, Figure $2 A$ ). Results persisted with excluding cardiac death due to causes other than SCD (RR $1.06,95 \%$ CI 0.69-1.62, $\mathrm{P}=0.80$, Figure 2B).

\section{Discussion}

In this systematic review and meta-analysis of five RCTs pooling data for 1,503 ICD recipients with NICM compared to 1,364 NICM receiving medical therapy, we demonstrate a clinically significant reduction in all-cause mortality (24\%) and SCD (60\%), but not cardiac death. The benefit of ICD for the prevention of all-cause mortality persisted despite robust sensitivity analyses, where we demonstrate that the use of ICDs drove the overall results.

The negative results of some of the individual RCTs are likely because of under-powering, which is where metaanalyses excel. By combining the results of multiple trials, the problem of power is overcome. The tradeoff is however that combining multiple trials brings in an element of heterogeneity that may impact our ability to interpret the results. Because of this limitation, it is important to apply rigorous statistical methods including sensitivity analyses to ensure that no one study is skewing the results.

Of all the studies examining the impact of ICD therapy compared to medical therapy on all-cause mortality, the DANISH trial stands out as the most negative. One important explanation of this finding is the fact that the medical therapy arm of the DANISH trial had $58 \%$ of patients who received CRT pacemakers. An interesting question that deserves further investigation is whether ICD therapy provides protection beyond medical therapy in the presence of CRT pacing. To date, there is no evidence from an RCT to confirm this statement. Although subgroup analyses are meant to be hypothesis generating; it is worth noting that the DANISH trial provided subgroup analyses with the outcome stratified according to CRT implantation status and showed no mortality benefit with ICD over medical therapy regardless if patients were in the groups who received CRT or not. Also, the COMPANION trial, which included a CRT-pacemaker and a CRT-defibrillator arm failed to show a difference between these two treatment strategies for the outcome of all-cause mortality. A recent meta-analysis (14) that included 19 studies (12,378 patients) showed that CRT-D patients had significantly lower mortality as compared to CRT-P patients (16.6\% vs. $27.1 \%$; $\mathrm{RR}=0.69,95 \%$ CI $0.62-0.76 ; \mathrm{P}<0.00001)$. However, when patients were divided into ICM and NICM, there was a non-statistically significant trend for benefit in patient with NICM receiving CRT-D as compared to CRT-P (HR 0.79, 95\% CI 0.61-1.02; $\mathrm{P}=0.07)$. Thus, future studies are needed to elucidate this important question of benefit of CRT-D as compared to CRT-P, particularly given the significant differences in size and cost between CRT pacemakers and defibrillators.

In addition, the DANISH trial had significantly better optimal medical therapy with ACE-I/ARB, Beta-blockers, and MRA. This may have contributed to diluting the mortality difference between the ICD and medical therapy arms of DANISH. This is all while keeping in mind that optimal medical therapy is a moving target with newer and potentially better therapies that could improve survival in NICM, further diluting the benefit of ICDs. The best example of this is the recently published PARADIGMHF trial which has shown that an Angiotensin ReceptorNeprilysin Inhibitors (i.e., Sacubitril/Valsartan combination) was superior to Enalapril in reducing the risk of death and heart failure admissions (15). Further investigation is needed to study the effect of the combination of Sacubitril/ Valsartan on the occurrence of SCD in patients with NICM.

Heart failure complicating NICM is a complex syndrome; therefore, the all-cause mortality and cardiac mortality outcomes in this population can be influenced by the burden of cardiac and non-cardiac comorbidities in these patients. In the two large trials which classified deaths during follow up into all-cause, cardiac and non-cardiac deaths (DANISH and DEFINITE), there were relatively high rates of cardiac deaths (due to causes other than SCD) and non-cardiac death rate. Given competing causes of death, this could be one possible explanation as why ICD therapy failed to reduce all causes mortality in both the DEFINITE and DANISH, despite the fact that ICD therapy was shown to still significantly reduce the rates of SCD. 
Another potential difference among the individual trials included in this meta-analysis may be the time from the onset of heart failure symptoms to ICD implantation. It is plausible that patients who remain in heart failure with reduced ejection fraction for a longer period of time despite optimal medical therapy tend to have higher event rates of events compared to patients with shorter time to ICD with or without CRT therapy. Unfortunately, these factors could not be corrected for in our present study. Also, a longer duration of follow up from the time of implantation of ICD to the conclusion of a study is likely to affect the outcome. As shown in our sensitivity analysis, limiting the analysis to studies with a follow up period $\leq 3$ years, showed a statistically significant reduction in all-cause mortality in patients undergoing ICD implantation as compared to medical therapy (RR 0.64, 95\% CI 0.47-0.87, $\mathrm{P}=0.004$ ). However, by limiting the analysis to DANISH and SCDHeFT which had a longer follow up, there was a trend toward benefit in ICD group that did not achieve statistical significance (RR 0.83, 95\% CI 0.67-1.02, $\mathrm{P}=0.07$ ). However, this result was driven by the magnitude of the DANISH trial, which makes it hard to draw conclusions with regards to the benefit of ICD in relation to follow up time. Further studies with similar long term follow up are needed to address this question. In the context of the older published trials, the DANISH trial showed us that we cannot continue practicing modern medicine based solely on older trials. As we glance into the future of primary prevention of SCD in NICM, there is a need for newer and pragmatic trials that address same questions with newer approaches, taking into account the evolution of cardiac therapy in general and incorporating more comprehensive risk assessment strategies using newer technology such as cardiac magnetic resonance, for example, to better risk stratify patients with NICM beyond just relying on the combination of symptoms and reduced ejection fraction as the only "criteria" for implanting ICDs.

\section{Limitations}

This is a meta-analysis of the published RCTs and therefore individual patient data were not available. The studies had slightly different designs, but were similar enough in their inclusion criteria to allow pooling of patient data, particularly that after assessment of the Inconsistency index $\left(\mathrm{I}^{2}\right)$ and the Q-statistic, there was no significant heterogeneity in any of the reported analyses. When considering the outcomes of cardiac death or SCD, competing causes of death, such as non-cardiac death, may impact the assessment of the event of interest. This is a major reason why we chose all-cause mortality as our primary outcome of interest for this analysis, since it is not subject to this limitation. In addition, for the outcome of SCD, it is often challenging to ascertain the exact cause of cardiac death, especially in patients assigned to the medical therapy arm. However, the benefit of ICD therapy in reducing SCD was demonstrated in multiple RCTs, and persisted through our sensitivity analysis.

\section{Conclusions}

Our pooled analysis demonstrates that ICD therapy reduces all-cause mortality and SCD in NICM patients, which is consistent with the recommendation of current published guidelines.

\section{Acknowledgements}

Dr. Saba received research grant support from Medtronic, Boston Scientific and St. Jude Medical. Dr. Jain received research grant support from Medtronic. Dr. Adelstein received research grant support from Medtronic.

\section{Footnote}

Conflicts of Interest: Dr. Saba received research grant support from Medtronic, Boston Scientific and St. Jude Medical. Dr. Jain received research grant support from Medtronic. Dr. Adelstein received research grant support from Medtronic. Other co-authors have no conflict of interest.

\section{References}

1. Dec GW, Fuster V. Idiopathic dilated cardiomyopathy. $\mathrm{N}$ Engl J Med 1994;331:1564-75.

2. Kannel WB, Wilson PW, D'Agostino RB, et al. Sudden coronary death in women. Am Heart J 1998;136:205-12.

3. Bänsch D, Antz M, Boczor S, et al. Primary prevention of sudden cardiac death in idiopathic dilated cardiomyopathy: the Cardiomyopathy Trial (CAT). Circulation 2002;105:1453-8.

4. Bristow MR, Saxon LA, Boehmer J, et al. Cardiacresynchronization therapy with or without an implantable defibrillator in advanced chronic heart failure. $\mathrm{N}$ Engl J Med 2004;350:2140-50.

5. Køber L, Thune JJ, Nielsen JC, et al. Defibrillator 
Implantation in Patients with Nonischemic Systolic Heart Failure. N Engl J Med 2016;375:1221-30.

6. Kadish A, Dyer A, Daubert JP, et al. Prophylactic defibrillator implantation in patients with nonischemic dilated cardiomyopathy. N Engl J Med 2004;350:2151-8.

7. Bardy GH, Lee KL, Mark DB, et al. Amiodarone or an implantable cardioverter-defibrillator for congestive heart failure. N Engl J Med 2005;352:225-37.

8. Writing Committee Members, Yancy CW, Jessup M, et al. 2013 ACCF/AHA guideline for the management of heart failure: a report of the American College of Cardiology Foundation/American Heart Association Task Force on practice guidelines. Circulation 2013;128:e240-327.

9. Ponikowski P, Voors AA, Anker SD, et al. 2016 ESC Guidelines for the diagnosis and treatment of acute and chronic heart failure: The Task Force for the diagnosis and treatment of acute and chronic heart failure of the European Society of Cardiology (ESC)Developed with the special contribution of the Heart Failure Association (HFA) of the ESC. Eur Heart J 2016;37:2129-200.

10. Liberati A, Altman DG, Tetzlaff J, et al. The PRISMA

Cite this article as: Masri A, Hammadah M, Adelstein E, Jain S, Saba S. Implantable cardioverter defibrillator in non-ischemic cardiomyopathy: a meta-analysis of randomized controlled trials. Cardiovasc Diagn Ther 2017;7(4):397-404. doi: 10.21037/ cdt.2017.06.06 statement for reporting systematic reviews and metaanalyses of studies that evaluate health care interventions: explanation and elaboration. J Clin Epidemiol 2009;62:e1-34.

11. DerSimonian R, Laird N. Meta-analysis in clinical trials. Control Clin Trials 1986;7:177-88.

12. Higgins JP, Green S. Cochrane Handbook for Systematic Reviews of Interventions Version 5.1.0. The Cochrane Collaboration, 2011. Available online: http://handbook. cochrane.org/

13. Begg CB, Mazumdar M. Operating characteristics of a rank correlation test for publication bias. Biometrics 1994;50:1088-101.

14. Barra S, Providência R, Tang A, et al. Importance of Implantable Cardioverter-Defibrillator Back-Up in Cardiac Resynchronization Therapy Recipients: A Systematic Review and Meta-Analysis. J Am Heart Assoc 2015;4. pii: e002539.

15. McMurray JJ, Packer M, Desai AS, et al. Angiotensinneprilysin inhibition versus enalapril in heart failure. $\mathrm{N}$ Engl J Med 2014;371:993-1004. 


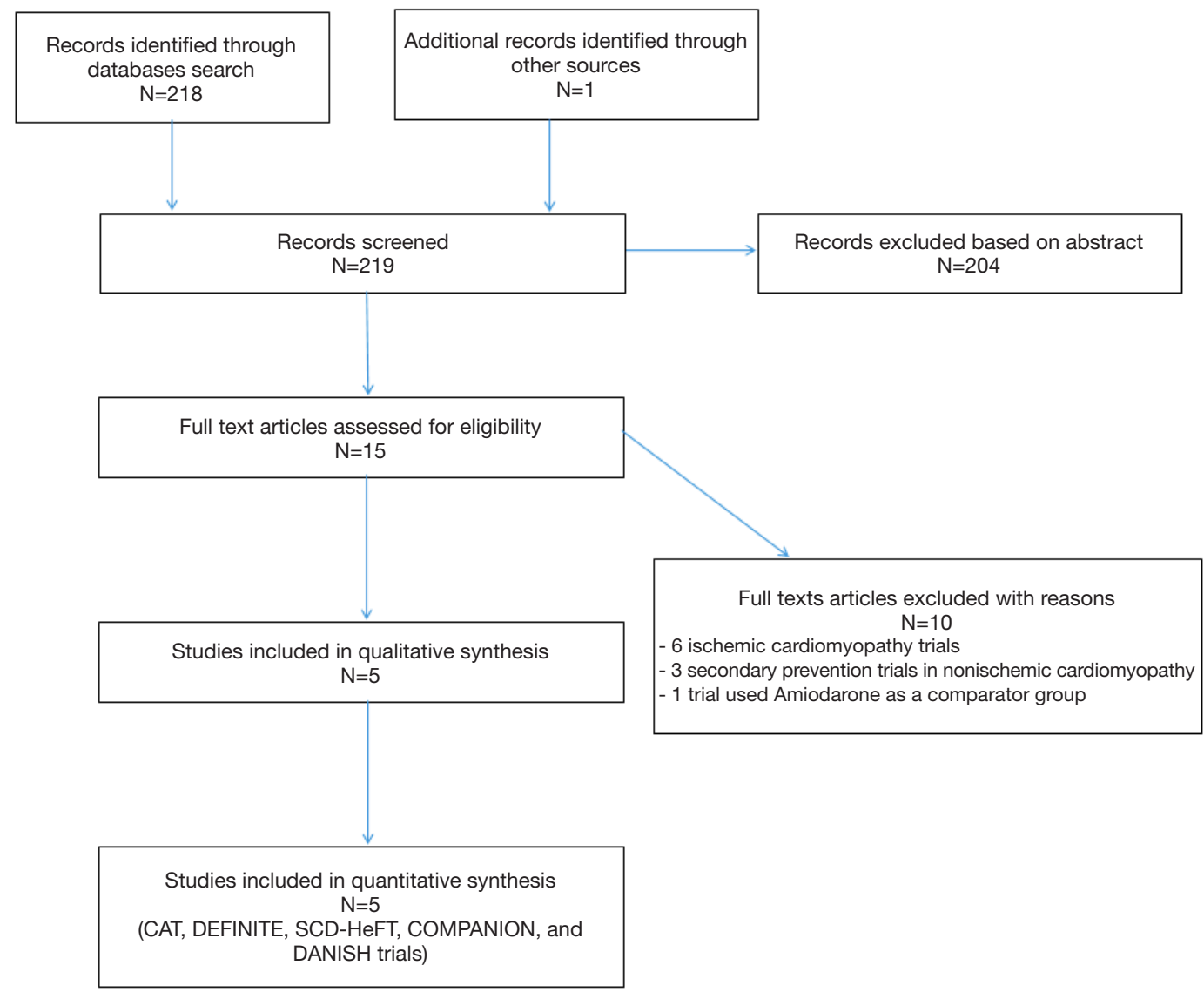

Figure S1 Diagram showing the search methodology, inclusion steps, and included articles. 


\begin{tabular}{|c|c|c|c|c|c|c|c|c|c|c|c|c|c|c|c|c|c|}
\hline RCT & Study design & Enrollment years & No. of patient enrolled & Age (years) & Male (\%) & QRS duration (msec) & LBBB (\%) & LVEF (\%) & NYHA class (\%) & DM (\%) & AF (\%) & ACE-I or ARB (\%) & Beta-blockers (\%) & MRA (\%) & Amiodarone (\%) & $\begin{array}{lll}\text { CRT (\%) } \\
\end{array}$ & Crossover to ICD (\%) \\
\hline CAT (NICM) & ICD vs. medical therapy & 1991-1997 & $50 / 54$ & $52 / 52$ & $86 / 74$ & $102 / 114$ & $23 / 37$ & $24 / 25$ & $\begin{array}{l}\text { II (66.7/64.1), } \\
\text { III (33.3/35.8) }\end{array}$ & NR & $20.4 / 11.3$ & $94 / 98.1$ & $4 / 3.7$ & NR & NR & $0 / 0$ & NR \\
\hline DEFINITE (NICM) & ICD vs. medical therapy & 1998-2002 & 229/229 & $58.4 / 58.1$ & $72.5 / 69.9$ & $114.7 / 115.5$ & $19.7 / 19.7$ & $20.9 / 21.4$ & $\begin{array}{l}\text { | (25.3/17.9), } \\
\text { || (54.2/60.7), } \\
\text { II (20.5/21.4) }\end{array}$ & $22.7 / 23.1$ & $22.7 / 26.2$ & $\begin{array}{l}\text { ACEI (83.8/87.3), } \\
\text { ARB (13.5/8.7) }\end{array}$ & $85.6 / 84.3$ & NR & $3.9 / 6.6$ & $0 / 0$ & 10 \\
\hline SCD-HeFT (NICM and ICM) $)^{\mu}$ & $\begin{array}{l}\text { ICD vs. medical therapy vs. } \\
\text { Amiodarone }\end{array}$ & $1997-2001$ & $829 / 847$ & $60.1 / 59.7^{*}$ & 77/77 & NR & NR & $24 / 25^{*}$ & $\begin{array}{l}\text { II (68/70), } \\
\text { III (32/30) }\end{array}$ & $31 / 32$ & $17 / 14$ & $94 / 98$ & $69 / 69$ & $20 / 19$ & $14 / 9.5$ & $0 / 0$ & $\mathrm{NR}^{*}$ \\
\hline COMPANION (NICM and ICM ${ }^{\mu}$ & $\begin{array}{l}\text { ICD/CRT vs. CRT vs. } \\
\text { medical therapy }\end{array}$ & 2000-2002 & $595 / 308$ & $66 / 68$ & $67 / 69$ & 160/158 & $73 / 70$ & 22/22 & $\begin{array}{l}\text { III (86/82), } \\
\text { IV (14/18) }\end{array}$ & $41 / 45$ & NR & $90 / 89$ & $68 / 66$ & $55 / 55$ & NR & 100/0 & 26 \\
\hline DANISH (NICM) & ICD vs. medical therapy & 2008-2014 & $556 / 560$ & $64 / 63^{*}$ & $73 / 72$ & $146 / 145$ & NR & $25 / 25$ & $\begin{array}{l}\text { II (53/54), } \\
\text { III (45/45), } \\
\text { IV (1/1) }\end{array}$ & $18 / 20$ & $24 / 20$ & 96/97 & 92/92 & $59 / 57$ & $6 / 6$ & $58 / 58$ & 4.8 \\
\hline
\end{tabular}

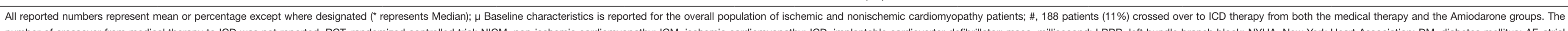

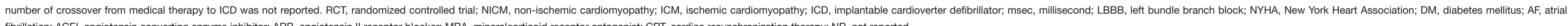

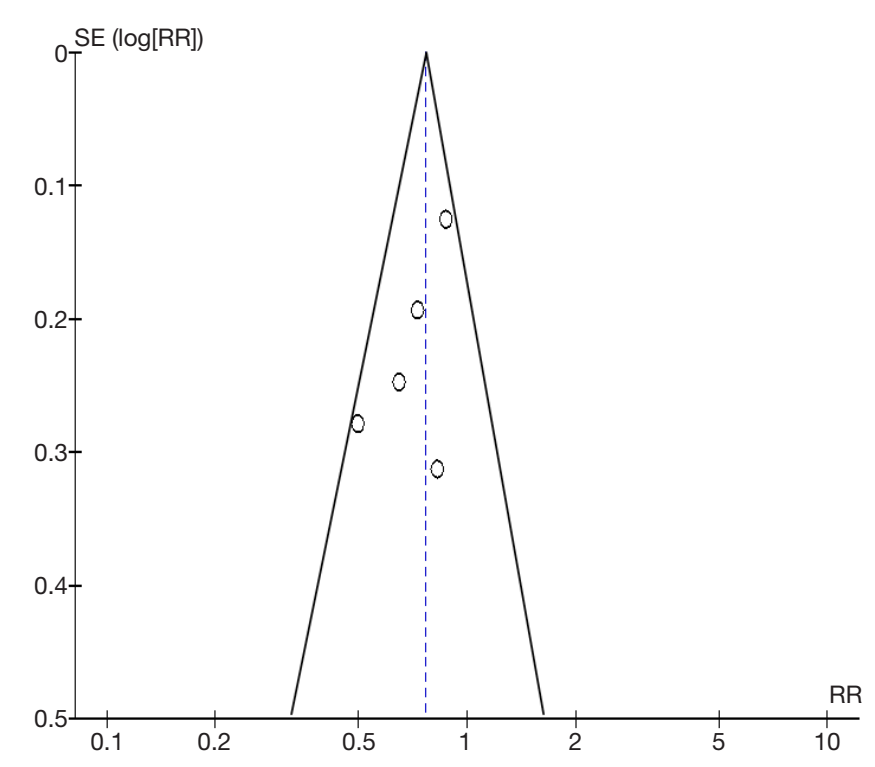

Figure S2 Funnel plot for the six randomized control trials evaluating all-cause mortality in patients receiving ICD versus medical therapy \pm Amiodarone. 


\begin{tabular}{|c|c|c|c|c|c|c|c|c|c|}
\hline Study or Subgroup & log[Risk Ratio] & SE & $\begin{array}{l}\text { ICD } \\
\text { Total }\end{array}$ & $\begin{array}{r}\text { Medical therapy } \\
\text { Total } \\
\end{array}$ & Weight & $\begin{array}{l}\text { Risk Ratio } \\
\text { IV, Random, } 95 \% \text { Cl }\end{array}$ & \multicolumn{3}{|c|}{$\begin{array}{c}\text { Risk Ratio } \\
\text { IV, Random, } 95 \% \mathrm{Cl}\end{array}$} \\
\hline CAT & -0.1863 & 0.3123 & 50 & 54 & $10.7 \%$ & $0.83[0.45,1.53]$ & & & \\
\hline COMPANION & -0.6931 & 0.2779 & 270 & 127 & $13.5 \%$ & $0.50[0.29,0.86]$ & & & \\
\hline DANISH & -0.1863 & 0.1829 & 234 & 237 & $31.1 \%$ & $0.83[0.58,1.19]$ & & & \\
\hline DEFINITE & -0.4308 & 0.2477 & 229 & 229 & $16.9 \%$ & $0.65[0.40,1.06]$ & & & \\
\hline SCD-HeFT & -0.3147 & 0.1931 & 398 & 394 & $27.9 \%$ & $0.73[0.50,1.07]$ & & & \\
\hline \multicolumn{3}{|l|}{ Total $(95 \% \mathrm{Cl})$} & 1181 & 1041 & $100.0 \%$ & $0.72[0.59,0.88]$ & & & \\
\hline \multicolumn{7}{|c|}{$\begin{array}{l}\text { Heterogeneity: } \operatorname{Tau}^{2}=0.00 ; \mathrm{Ch}^{2}=2.71, \mathrm{df}=4(\mathrm{P}=0.61) ; \mathrm{I}^{2}=0 \% \\
\text { Test for overall effect: } Z=3.25(P=0.001)\end{array}$} & $\frac{1}{0.2}$ & $0.5 \quad 1$ & $12 \frac{5}{2} \quad 5$ \\
\hline
\end{tabular}

Figure S3 All-cause mortality in ICD vs. medical therapy groups with the DANISH trial only reporting on patients with no CRT implantation.

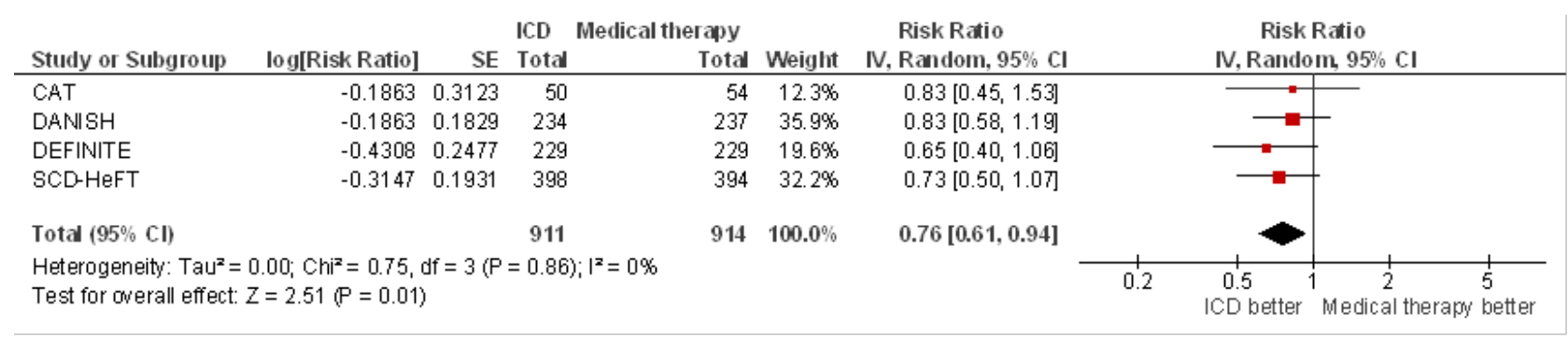

Figure S4 All-cause mortality in ICD vs. medical therapy groups excluding the COMPANION trial and including DANISH trial patients with no CRT implantation. 